\title{
ATTENTION AND AMBLYOPIA* AN ELECTRO-ENCEPHALOGRAPHIC APPROACH TO AN OPHTHALMOLOGICAL PROBLEM
}

BY

\author{
A. T. M. VAN BALEN AND H. E. HENKES \\ The Eye Clinic, Rotterdam, Netherlands
}

IN a previous paper (van Balen and Henkes, 1960) we indicated the possibility of recording the response of the occipital lobe to light-stimulation in man. The use of Dawson's superimposition method together with the mathematical integration method enabled us to record the responses in all normal subjects.

The specific response of the visual cortex consists of four different components: a small surface-positive wave $a$, followed by a somewhat larger negative wave $b$, then by the main positive deflection $c$ and a smaller negative deflection $d$ (van Balen and Henkes, 1960, Fig. 5). The positive deflection $c$ consists of two components: $c^{1}$ and $c^{2}$. A study of the specific response to light stimulation of varying intensities and at different levels of dark adaptation revealed the correspondence of the amplitude of the $c^{1}$-wave with the level of activity of the photopic mechanism, and of the amplitude of the $c^{2}$ wave with the level of activity of the scotopic mechanism.

The photopic and scotopic mechanisms, however, are concepts of the physiology of the retina and coincide more or less with the anatomical concepts of cones and rods.

In the ganglion cell layer of the retina a regrouping occurs. Foveal cones in monosynaptic units form the foveal system; peripheral cones and rods in polysynaptic units form the extra-foveal system. Anatomically the foveal and extra-foveal systems can be distinguished in all parts of the visual system. In the visual cortex (striate area), for instance, a foveal ("macular") and extrafoveal ("extra-macular") projection field can be distinguished. These data lead one to expect the separate representation of the foveal and extra-foveal systems in the occipital response.

\section{Representation of the Foveal and Extra-foveal Systems in the OCCI- PITAL LOBE RESPONSE}

In some of our subjects the $c^{1}$-wave could be led off only from the posterior part of the scalp in the occipital region, while the $c^{2}$-wave was led off from both the anterior and posterior parts (Fig. 1, opposite). This corresponds with the position of the foveal projection field in the posterior part of the striate area.

* Received for publication March 3, 1961. 

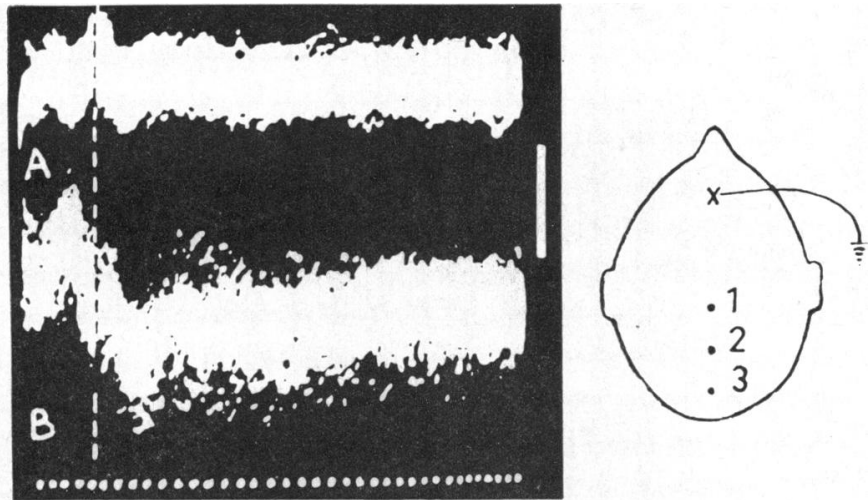

FIG. 1.-Recording of normal human occipital electro-encephalogram after light stimulation by Dawson's superimposition technique.

Energy of stimulator flash: $4 / 3$ Joule.

Distance from eye to stimulator: $24 \mathrm{~cm}$.

Background illumination: 100 Lux.

Voltage calibration: $20 \mu \mathrm{V}$.

Time calibration: 50 cycles per second.

Vertical dotted line: at $100 \mathrm{msec}$. after stimulus.

A : Leading off between Electrodes 1 and 2.

B: Leading off between Electrodes 2 and 3 .

Electrodes located towards the rear (2 and 3) are able to record a response in which an early positive wave is present that does not occur in records taken from the anterior electrodes.

The hypothesis according to which the $c^{1}$-wave represents foveal activity and the $c^{2}$-wave extra-foveal activity is supported by findings in patients with total colour blindness, in whom (Fig. 2) the shape of the specific occipital response which follows high-intensity stimulation in light adaptation is essentially the same as that which follows low-intensity stimulation in dark adaptation. Moreover, the $c$-wave of the occipital response in these cases has the same culmination time in both photopic and scotopic stimulus conditions. The

FIG. 2.-Record of occipital electroencephalogram in a case of total colour blindness, using Dawson's superimposition and a mathematical integration technique.

Energy of stimulator flash:

(1) $4 / 3$ Joule, - upon a background illumination of 100 Lux.

(2) $1 / 12$ Joule in dark adaptation for 10 minutes.

Distance from eye to stimulator $27 \mathrm{~cm}$. The $c$-waves are essentially similar under photopic conditions (1) and scotopic conditions (2). Culmination time: $110 \mathrm{msec}$. after stimulus.

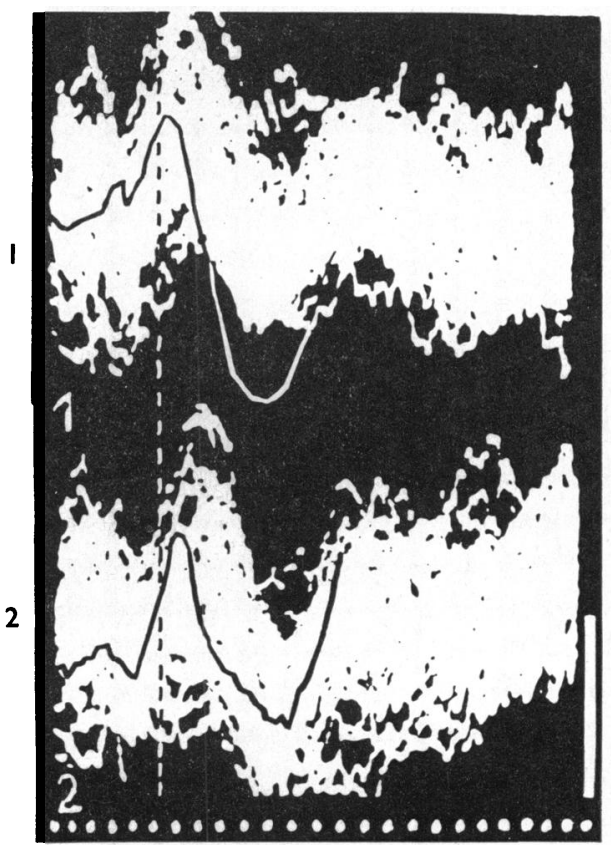


$c$-wave culminates at a point between the culmination times of the $c^{1}$ and $c^{2}$-waves of the normal occipital electro-encephalogram. In total colour blindness, the $c$-wave seems to represent a system (presumably the extra-foveal system) that is able to react under both photopic and scotopic conditions. The activity of the foveal system (normally represented in the $c^{1}$-wave) seems to be abolished in these cases and the loss of the inhibitory effect normally exerted by the foveal cones on the combined peripheral cones and rods (Granit, 1947) seems to allow the remaining $c$-wave to culminate earlier than a normal $c^{2}$-wave. Arguments for the existence of such a two-fold "scotopic" mechanism in cases of total colour blindness are given by Hecht, Shlaer, Smith, Haig, and Peskin (1948), Sloan (1954), Peskin (1954) and Yonemura and Ishisaka (1955), and in normal individuals by Jayle, Ourgaud, Lozivit, and Aubert (1957), Ronchi and Bittini (1958), and Ingvar (1959).

The hypothesis mentioned above is supported by experiments with the influence evoked by the subject's attention on the form of the evoked potential of the occipital cortex.

\section{INFLUENCE OF ATTENTION ON THE FORM OF THE OCCIPITAL RESPONSE}

In the present study an effort was made to direct the subject's attention to the light stimulus by presenting optotypes during light stimulation. These optotypes had to be deciphered, they were not distinguishable in the interval between two successive stimuli. In parallel experiments light stimuli of the same intensity were offered without presenting optotype, while attention was distracted by a simultaneous auditory stimulation.

Comparison of the responses "with attention" and "without attention" (see Fig. 3, opposite) reveals a change in the relation of the $c^{1}$ - and the $c^{2}$-wave. The $c^{1}$-wave appears clearly in the attentive state using lowintensity light flashes, but in the non-attentive state responses to the same intensity of flash show a $c^{2}$-wave only. In responses to high-intensity stimuli in the attentive state, the $c^{1}$-wave exceeds the $c^{2}$-wave to a greater extent than in responses in the non-attentive state. In some of our subjects, more particularly while using high-intensity stimuli, the influence of attention is seen more in a reduction of the $c^{2}$-wave than in an increase of the $c^{1}$-wave (see Fig. 4, opposite, and Fig. 5, overleaf).

Attention seems to favour the development of the $c^{1}$-wave (representing the activity of the foveal system) at the cost of the $c^{2}$-wave (representing the activity of the extra-foveal system). A presumed influence of the attention in the electroretinogram (depression of the amplitude of the positive $b$-wave: Fig. 6, overleaf) disappeared during pupillary dilatation (Fig. 5, overleaf).

\section{OcCipital Response to Stimulation of Amblyopic Eyes}

The difference between the amplitudes of the $c^{1}$ - and $c^{2}$-waves in the responses of the normal eye "with attention" and "without attention" is 
(I)

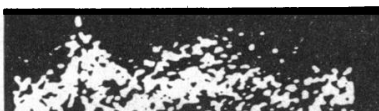

(2)

(3)

(4)

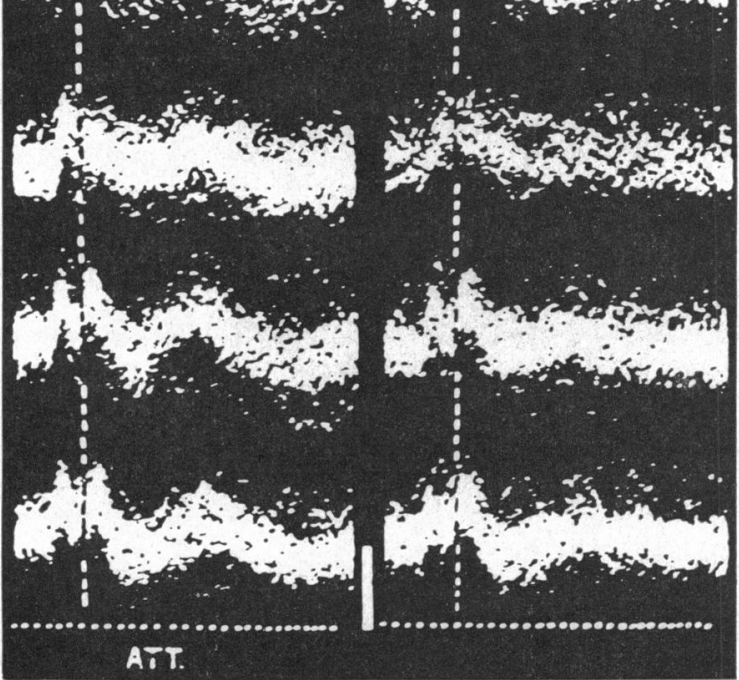

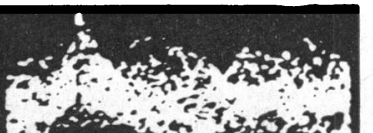
tion upon evoked potential.

Left: attentive fixation ("ATT") of light source.

Right: fixation without attention.

Light adaptation (background illumination 100 Lux).

Energy of flash

(1) $1 / 12$ Joule + Neutral densityfilter (Factor 8).

(2) $1 / 12$ Joule

(3) $1 / 3$ Joule

(4) $4 / 3$ Joule

Distance between eye and light source: $60 \mathrm{~cm}$.

Light source presented behind a screen with groundglass window of $7 \mathrm{~cm}$. diameter (Density Factor 6).

Calibrations: as in Fig. 1. 


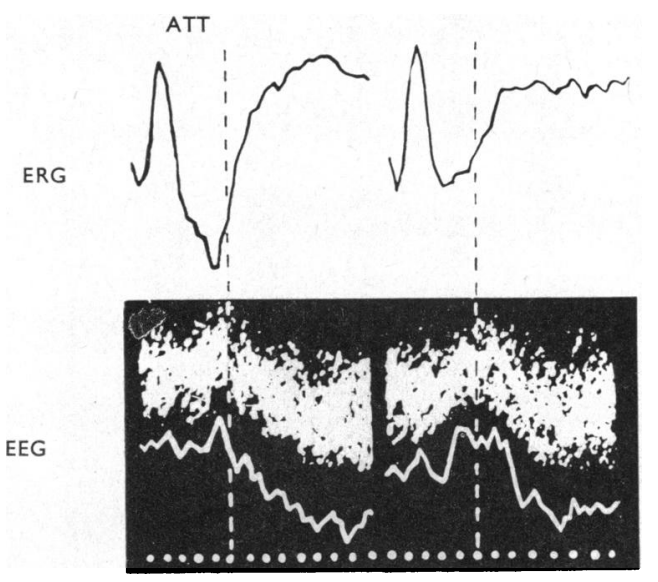

FIG. 5.-Influence of attention upon the electroretinogram and the occipital electro-encephalogram. Occipital electro-encephalogram recorded by Dawson's superimposition technique and occipital electro-encephalogram and electroretinogram by the mathematical integration method.

Energy of flash: $4 / 3$ Joule.

Distance between subject's eye and light source: $24 \mathrm{~cm}$.

Artificial pupillary dilatation.

Light adaptation (background illumination: 100 Lux).

In the attentive state (ATT) the electro-encephalogram response shows an early positive wave $\left(c^{1}\right)$ culminating at $80 \mathrm{msec}$, and in the inattentive state the response shows a broad positive wave culminating from 80 to $130 \mathrm{msec}$., suggesting the presence of $c^{1}$ and $c^{2}$ components. The electroretinogram does not differ essentially in the two conditions.

A

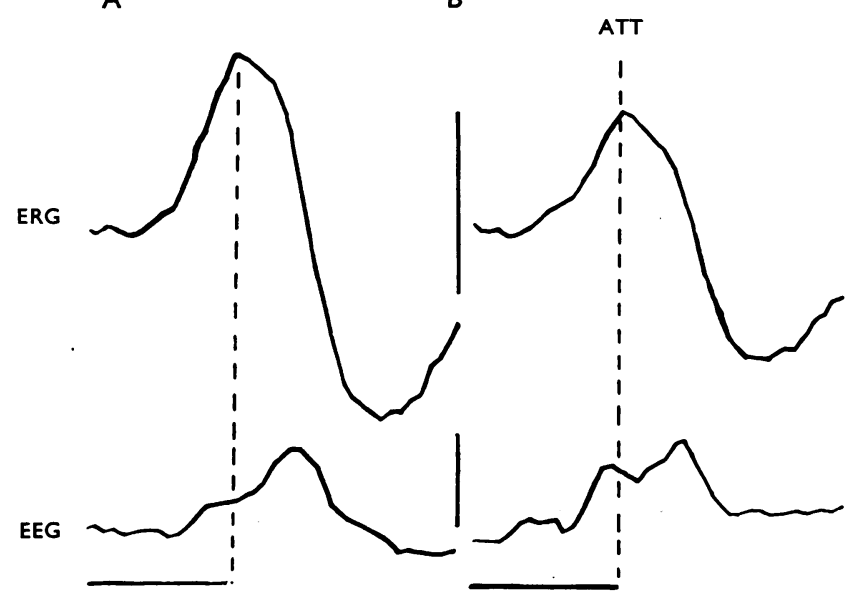

FIG. 6.-Influence of attention upon electroretinogram and occipital electro-encephalogram, recorded by the mathematical integration method for the left eye, the right eye being occluded.

Energy of flash: $1 / 6$ Joule.

Dark adaptation.

Other conditions same as in Fig. 3.

Voltage calibration $20 \mu \mathrm{V}(\mathrm{ERG})$ and $10 \mu \mathrm{V}$ (EEG).

Time calibration: $100 \mathrm{msec}$.

In the attentive state (ATT) the electro-encephalogram shows a double $c$-wave ( $c^{1}$ and $\left.c^{2}\right)$. In the inattentive state only one $c$-wave $\left(c^{2}\right)$ is present. Contrary to the findings shown in Fig. 5 , the amplitude of the $b$-wave of the electroretinogram is smaller in the attentive state.

This difference disappeared after artificial mydriasis. 
similar to the differences between the responses to stimulation of the normal and the amblyopic eye in the same subject.

The $c^{1}$-wave of the amblyopic eye may be somewhat smaller than that of the normal eye (Fig. 7), or the $c^{2}$-wave response seen of the normal eye may be reduced in comparison to that in the amblyopic eye (Fig. 8).

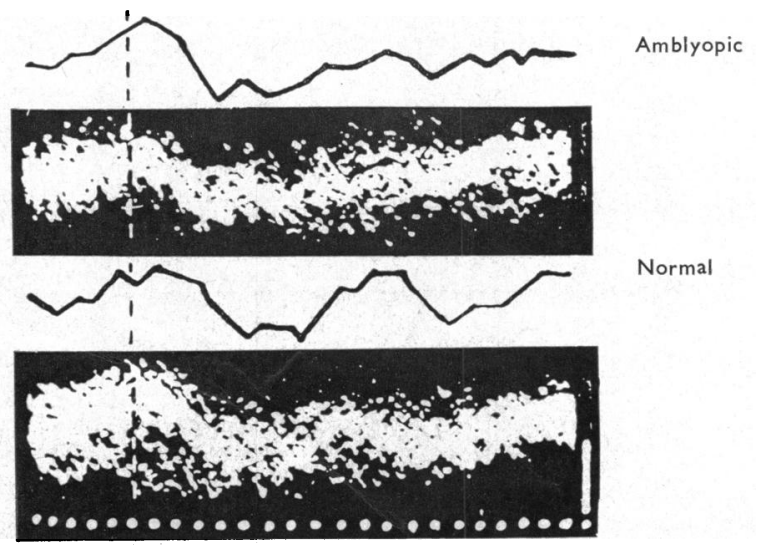

FIG. 7.-Evoked potential of occipital lobe after stimulation of amblyopic eye and normal eye in a patient suffering from squint amblyopia (visual acuity $0 \cdot 1$, with central fixation).

Energy of flash: $4 / 3$ Joule.

Recording conditions and calibration as in Fig. 3.

The ascending slope of the $c$-wave complex for the normal eye is steeper than for the amblyopic eye, suggesting the presence of an early component $\left(c^{1}\right)$ in the normal response. After-discharge (latency $240 \mathrm{msec}$., duration $120 \mathrm{msec}$.) more pronounced in normal eye.

Normal

Amblyopic

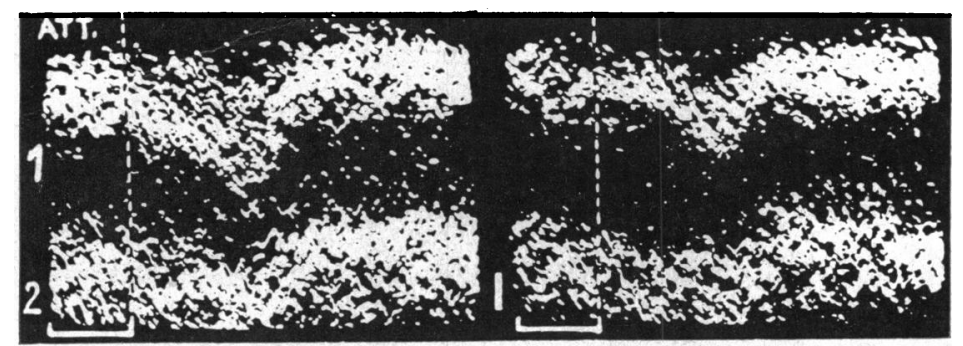

FIG. 8.-Evoked potential in a patient suffering from squint amblyopia, in the attentive state (1) when the patient could read Snellen's letters during the presentation of the light flash, and in the inattentive state (2).

Recording conditions same as in Fig. 7.

In the attentive state (Tracing 1), the evoked response of the normal eye differs from that of the amblyopic eye.

The normal tracing deflects to negativity immediately after the $100-\mathrm{msec}$. line, but the amblyopic response does not begin to deflect to negativity until $160 \mathrm{msec}$. This suggests that the depression of the $c^{2}$-wave is more pronounced in the "normal" than in the "amblyopic" response.

In the inattentive state (Tracing 2), the responses of the normal and amblyopic eye seem to be identical.

From an electrophysiological point of view, squint amblyopia may very well be compared to a state of vision "without attention", at least as far as the form of the specific occipital response is concerned. 


\section{Influence OF AtTention on the After-Discharge of THE OCCIPITAL LoBe} RESPONSE

Like the evoked responses of other sensory systems, the occipital lobe response shows an after-discharge. Bartley (1934) mentioned after-discharges of the alpha-wave type. We were able to record an after-discharge of this type in a number of subjects, but in others the specific response was followed by only one single after-discharge, consisting of one wave of approximately $200 \mathrm{msec}$. duration.

Attention increases the amplitude of both types of after-discharge: the alpha-wave type (Fig. 9) and the single after-discharge wave (Fig. 3).

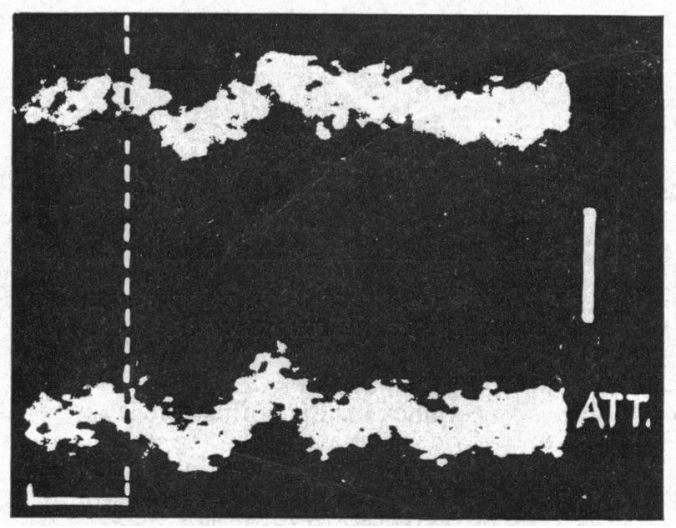

Fig. 9.-Influence of attention upon the after-discharge of evoked potential.

Recording conditions same as in Fig. 7.

Technique of alternating responses recorded in the attentive state (ATT) and the inattentive state as in Fig. 4.

In the attentive state (ATT) the $c^{2}$-wave is smaller and the after-discharge of three waves (duration approx. $100 \mathrm{msec}$.) is of higher amplitude than in the inattentive state.

The influence of attention on the height of the after-discharge wave seems to be correlated with the influence of attention on the $c^{1}: c^{2}$ ratio in the specific response itself. The influence on the after-discharge is, however, less constant and usually less marked.

Our study revealed that an after-discharge of the alpha-wave type is found in subjects who show a considerable amount of spontaneous alpha activity, whereas the single after-discharge wave of approximately $200 \mathrm{msec}$. duration is found in subjects exhibiting only a sporadic spontaneous alpha-activity or none at all. In both groups of subjects some occipital lobe responses may be recorded in which no after-discharge is detected (Figs 1 and 4).

\section{Discussion}

In view of the anatomical and (electro)physiological data, it seems plausible to accept that, in the occipital lobe response to light stimulation, the $c^{1}$ - and 
$c^{2}$-waves represent the foveal and the extra-foveal systems respectively. This hypothesis does not contradict that of the representation of the photopic and scotopic mechanism in the $c^{1}$ - and $c^{2}$ - waves of the occipital response (van Balen and Henkes, 1960), but should be regarded as the consequence of it.

Several experiments dealing with the influence of attention on the specific sensory responses, including the evoked response of the visual cortex (Gellhorn, Koella, and Ballin, 1954), are described in the literature. This influence is presumably mediated by way of the reticular formation (R.F.) of the brainstem (Magoun, 1956). This system can be activated through both the ascending and descending pathways, the latter originating in various cortical regions. The influence of attention and R.F. activation upon the evoked responses has been described as an increase of the amplitude of the response. Our experiments stress the importance of the shift in the relation between the foveal and the extra-foveal systems which is expressed in the $c^{1}: c^{2}$ ratio.

Amblyopia should probably be regarded as a loss of R.F. activation, so that it may be the result of inattentiveness rather than of suppression.

Since amblyopia is unilateral, the point where "attention" intervenes must, obviously, be located in a part of the visual pathway, situated distally from the optic chiasma. We have been able to record the influence of attention on the amplitude of the $b$-wave of the electroretinogram, but we have found that this phenomenon disappears in pupillary dilation. In consequence we had to locate the point where attention intervenes between the origin of the electroretinogram and the optic chiasma, i.e. in the ganglion cell layer of the retina and/or the internal part of the bipolar layer. These layers are known to contain horizontal connexion cells which are able to mediate a regulating impulse between the foveal cones and the extra-foveal system which is composed of the cones and rods (Polyak, 1957).

The central regulation of the activity of these layers may be operated by way of centrifugal fibres in the optic nerve. Granit (1955) and Dodt (1956) demonstrated the presence of such fibres by electrophysiological methods.

The physiological basis of visual attention seems to consist in an activation of the foveal system at the expense of the activity of the extra-foveal system. This regulation may originate in the reticular formation of the brainstem and is mediated by way of the centrifugal fibres of the optic nerve to the retina. In a state of inattention, in physiological suppression, and in cases of squint amblyopia, this mechanism fails. This hypothesis conforms with our present knowledge of amblyopia. The relative central scotoma, the decreased pupillary reflex (Harms, 1938), and the decreased critical frequency of flicker fusion in the foveae of amblyopic eyes (Feinberg, 1956) are all signs of decreased activity of the foveal system.

The inquiries of von Noorden and Burian (1959) into the visual acuity of amblyopic eyes at various levels of illumination, and those of Oppel (1960) 
into dark adaptation, also seem to support the idea that the activity of the foveal system is decreased in cases of amblyopia.

The treatment of amblyopia by stimulating the fovea (Bangerter) or by depressing the activity of the extra-foveal system (Cüppers) can find its theoretical basis in this hypothesis.

\section{Summary}

The $c^{1}$ - and $c^{2}$-waves of the evoked response of the occipital lobe presumably represent the activity of the foveal system and the extra-foveal system respectively. This hypothesis is based upon anatomical data and is confirmed by the experiments described in this paper. The $c^{1-}$ and $c^{2}$-waves show topographical differences correlated with anatomical data. In achromatopsia the remaining $c^{2}$-wave still has the characteristics of a two-fold mechanism, suggesting the existence of combined activity of peripheral cones and rods (i.e. the extra-foveal system). Attentive fixation of the light stimulus enhances the activity of the foveal system at the expense of the activity of the extra-foveal system. This enhancement finds its expression in a shift in the ratio of the amplitudes of the $c^{1}$ - and $c^{2}$-waves. From the electrophysiological point of view, amblyopia due to squint may be regarded as vision without attention.

The possible role of the reticular formation of the mid-brain in connexion with attention and amblyopia is discussed.

\section{REFERENCES}

Balen, A. T. M. van, and Henkes, H. E. (1960). Brit. J. Ophthal., 44, 449.

Bartley, S. H. (1934). Amer. J. Physiol., 108, 397.

DodT, E. (1956). J. Neurophysiol., 19, 301.

FEINBERG, I. (1956). Amer. J. Ophthal., 42, 473.

Gellhorn, E., Koella, W. P., and Ballin, H. M. (1954). J. Neurophysiol., 17, 14.

GRANIT, R. (1947). "Sensory Mechanisms of the Retina". Oxford University Press, London. (1955). J. Neurophysiol., 18, 388.

Harms, H. (1938). v. Graefes Arch. Ophthal., 138, 149.

Hecht, S., Shlaer, S., Smith, E. L., Haig, C., and Peskin, J. C. (1948). J. gen. Physiol., $31,459$.

INGVAR, D. H. (1959). Acta physiol. scand., 46, Suppl. 159.

Jayle, G. E., Ourgaud, A. G., Lozivit, P., and Aubert, L. (1957). Ann. Oculist. (Paris), 190, 213.

Magoun, H. W. (1956). In "Nerve Impulse: Transactions of the 5th Conference, 1954, p. 11. Josiah Macy, Jr., Foundation, New York.

Noorden, G. K. von, and Burian, H. M. (1959). A.M.A. Arch. Ophthal., 61, 533; 62, 396.

OPPEL, O. (1960). Klin. Mbl. Augenheilk., 136, 563.

Peskin, J. C. (1954). J. appl. Physiol., 6, 661.

PolyaK, S. (1957). “The Vertebrate Visual System”. University of Chicago Press.

Ronch, L., and BitTINI, M. (1958). Atti Fond. G. Ronchi, 13, 538.

SLOAN, L. L. (1954). J. opt. Soc. Amer., 44, 117.

YonemuRA, D., and IshisaKa, N. (1955). Tohoku J. exp. Med., 62, 377. 